\title{
Evaluation of the Effect of Bone Grafts in Different Sizes and Forms Used During Alveolar Split Osteotomy on the Implant Stability
}

\author{
Ugur Mercan ${ }^{1,}$ *, Suheyla Kaya ${ }^{2}$ \\ ${ }^{1}$ Department of Oral and Maxillofacial Surgery, Faculty of Dentistry, Okan University, Istanbul, Turkey \\ ${ }^{2}$ Department of Periodontology, Faculty of Dentistry, Okan University, Istanbul, Turkey
}

Email address:

dr.umercan@yahoo.com (U. Mercan), shylakaya@gmail.com (S. Kaya)

${ }^{*}$ Corresponding author

\section{To cite this article:}

Ugur Mercan, Suheyla Kaya. Evaluation of the Effect of Bone Grafts in Different Sizes and Forms Used During Alveolar Split Osteotomy on the Implant Stability. International Journal of Clinical Oral and Maxillofacial Surgery. Vol. 6. No. 2, 2020, pp. 34-39.

doi: $10.11648 /$ j.ijcoms.20200602.13

Received: June 17, 2020; Accepted: July 3, 2020; Published: July 13, 2020

\begin{abstract}
Objective: To compare the effects of filling bone gap between implants with bone graft materials of different sizes and forms of primary stability after alveolar split osteotomy process. Materials and Methods: Materials and Methods: Fourty fresh ribs were divided into four main groups. The groups created are based on the xenograft material of different sizes and forms; group Putty (P): Group applied Putty (form) graft (granule size $0.25-1 \mathrm{~mm}$ ), group Small (S): Group applied Small particle graft (granule size $0.25-1 \mathrm{~mm}$ ) group, group Large (L): Group applied Large particle graft (granule size 1- $2 \mathrm{~mm}$ ), control group (C): classified as group where no bone graft was applied. Bone expansion was performed using piezosurgery. In all groups, two implants were inserted into the each rib after alveolar crest expansions. The gap between implants (outside the control group) was filled with bone graft materials of different forms and sizes (large, small, putty). The primary stability values of the implants were measured with in the direction of the bucco-lingual (BL) and mesio-distal (MD) by the ISQ (implant stability quotient) and compared between groups. Results: The primary stability values measured in the BL direction are higher than the primary stability values measured in the MD direction and values are statistically significant difference. There is no statistically significant difference between the 1st and 2nd implants in each group (C, P, S, L) for their measured values in both BL direction and MD direction. Conclusion: According to the ISQ values used to evaluate primary stability, it was found that the graft materials of different sizes used in our study did not cause a significant difference for the primary stability of the implants.
\end{abstract}

Keywords: Bone Graft, Alveolar Ridge Split, Implant, Primary Stability

\section{Introduction}

In the place of teeth lost for various reasons, dental implant treatments, which support chewing and speech functions and help make dental aesthetic restorations, have been used in dentistry for many years [1]. Loss of soft and hard tissue occurs around teeth lost due to trauma or infection [2]. Defects due to significant hard tissue loss may not allow ideal placement of dental implants. Various bone augmentation procedures are performed in cases of insufficient bone for dental implant placement [1]. Alveolar split osteotomy is one of the simple, effective, and reliable surgical techniques used to expand the bone in a horizontal direction [3, 4]. For alveolar split osteotomy, various instruments such as fissure burs, chisels and hammer or piezo surgery are used [5]. With piezo surgery, alveolar split osteotomy has become easier, safer, and less complicated [6, 7]. Compared with other instruments, the most important advantage of piezo surgery is the low risk of damaging anatomically important formations [6-8]. Generally, in alveolar split osteotomy, vertical incisions in the crest and bone are made using piezo surgery, and chisels and hammers are used for horizontal alveolar enlargement [6-8]. Alveolar split osteotomy can be performed as single or double-stage surgery. In a single-stage surgical procedure, implants are placed in the same session as bone expansion, and bone graft 
materials are placed in the space between. In two-stage surgery, alveolar enlargement is performed and augmentation is provided with a graft. Implants are placed after mature bone is obtained [3, 5]. Studies emphasized that interpositionally placed grafts increased implant stability and positively affected osseointegration in two-stage alveolar split osteotomy [3]. Autogene grafts, allografts, xenografts, and alloplastic graft materials are used in alveolar split osteotomy [9]. Primary stability is called the stiffness that occurs after the first contact between bone and implant, and is one of the most important factors affecting the osseointegration process and clinical success of dental implants [10]. The concept of primary stability is called secondary stability in the process of osseointegration [11]. Although the concept of primary stability is generally described as the mechanical interaction of bone and implant relationship, it is known that primary stability is influenced by bone quality and amount, implant design, and the surface properties (shape, geometry, length, diameter, surface) of the implants [11-13]. There is no contact of the implants with the bone in the mesio-distal direction of the implants placed in the cavities formed as a result of bone dilation performed by alveolar split osteotomy. In studies of these bone defect models, it has been shown that the bone-implant relationship is increased with bone graft materials of various sizes and forms $[3,9]$. There is insufficient research on how such bone graft materials used to fill defects that occur after split osteotomy after implant placement affect the primary stability of the implant. Resonance frequency analysis (RFA) is a non-invasive method that can be used during and after surgical procedures to evaluate primary and secondary stability [11]. With this device, which can measure in terms of implant stability quotient (ISQ) with Osstell Mentor (Osstell $^{\mathrm{TM}}$, Integration Diagnostics, Gothenburg, Sweden), values are graded between 1-100. It can also be used to evaluate the osseointegration process because it is noninvasive [14]. The aim of this study was to evaluate the effects of filling bone gaps/cavities between implants with bone graft materials of different sizes and forms on primary stability (implant stiffness) after the bone expansion process (alveolar split osteotomy).

\section{Materials and Methods}

\subsection{Specimen Preparation and Stability Measurements}

In this in-vitro study, 40 fresh sheep ribs were used, with which four $(n=10)$ main groups were formed. In order to provide uniform test conditions, ribs with the same cortical layer thickness $(3 \mathrm{~mm})$ were selected, which were compatible with Type II bone according to the Lekholm and Zarb classification [15]. The groups created were based on xenograft materials of different sizes and forms; putty (group P): a putty graft was used (granule size $0.25-1 \mathrm{~mm}$ ) (Geistlich Bio-Oss Pen ${ }^{\circledR}$, Geistlich, New Jersey, United States); small (group S): small particle grafts were used (granule size 0.25-1 $\mathrm{mm}$ ) (Geistlich Bio-Oss ${ }^{\circledR}$, Geistlich, New Jersey, United
States); large (group L): large particle grafts were used (granule size 1-2 mm) (Geistlich Bio-Oss Pen ${ }^{\circledR}$, Geistlich, New Jersey, United States), controls (group C): no bone graft was used (Figure 1). The distance between the two implants $(3+4+3)$ was calculated as $10 \mathrm{~mm}$ for placing two implants on the ribs, enough to place three implants with a diameter of 3.5 $\mathrm{mm}$ to mimic multiple tooth deficiencies. Distances to allow vertical osteotomy $1.5 \mathrm{~mm}$ lateral to both implant slots were marked (Figure 1). In accordance with these distances to the crest of the rib $(1.5+3.5+3+4+3+3.5+1.5 \mathrm{~mm}) 20-\mathrm{mm}$ (Figure 2) horizontal and $10-\mathrm{mm}$ long vertical osteotomies were performed. The first cuts required for alveolar split osteotomy were performed to all ribs using piezo surgery, and chisel osteotomies for alveolar enlargement. After the alveolar crest expansion was completed in both groups, implant drilling was performed to provide a $1.5 \mathrm{~mm}$ distance between the vertical osteotomy line and implant. First and second implants were inserted after the drilling was completed to accommodate the $3.5-\mathrm{mm}$ diameter (NobelActive $\AA$, Nobel Biocare, Gothenburg, Sweden) and 8-mm long implant. The space between implants (outside the control group) was filled with the various xenograft materials (large, small, putty). The primary stability values of the implants were bucco-lingual (BL) and mesio-distal (MD) (Figure 2), by measuring the ISQ three times with the Osstel device and their averages were taken by the same physician and compared between the groups.

\subsection{Statistical Analysis}

The IBM SPSS Statistics 22 (SPSS IBM, Turkey) program was used for the statistical analysis of the findings obtained in this study. The Shapiro-Wilk test was used to determine whether the parameters were normally or non-normally distributed, and they were found to be normally distributed. One-way analysis of variance (ANOVA) was used for intergroup comparisons of parameters, and Student's t-test was used for comparisons between directions and implants. The level of significance was determined as $\mathrm{p}<0.05$.

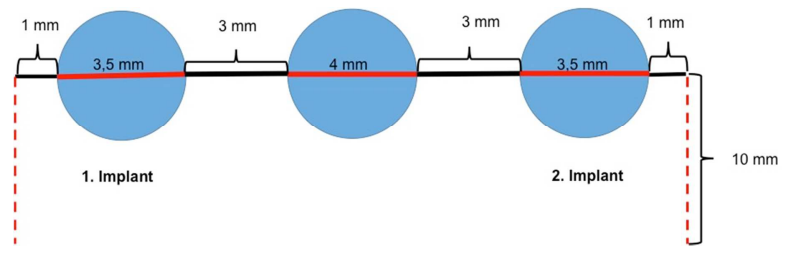

Figure 1. This schema shows the planned $(1.5+3.5+3+4+3+3.5+1.5$ mm) $20 \mathrm{~mm}$ horizontal and $10 \mathrm{~mm}$ long vertical osteotomy line and implant locations.

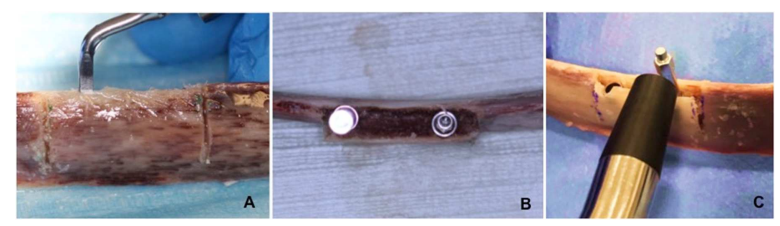

Figure 2. A; Alveolar split osteotomy were applied to all subjects by using piezosurgery. B; After the alveolar crest expansion was completed first and second implants were inserted. C; The primary stability values of the implants were measured by Osstell device. 


\section{Results}

When both the values of the first and second implants in all groups were measured in the BL direction, and the values measured in the MD direction were compared between the groups (groups $\mathrm{C}, \mathrm{P}, \mathrm{S}, \mathrm{L}$ ), no statistically significant difference was observed (Table $1,1 p)(p>0.05)$. When the ISQ values measured in the BL and MD directions were compared with each other, the values measured in the BL direction were statistically significantly higher than those in the MD direction $(p<0.001)$. Similarly, a statistically significant difference $(p<0.001)$ was found between the groups $(C, P, S, L)$ in the second implants in ISQ values measured in terms of BL and MD. The ISQ values of the second implant measured in the $\mathrm{BL}$ and $\mathrm{MD}$ directions were compared and the values measured in the BL direction were statistically significantly higher than the values in the MD direction (Table 1, 2p) $(\mathrm{p}<0.001 ; \mathrm{p}<0.05)$. There was no statistically significant difference between the first and second implants in each group $(\mathrm{C}, \mathrm{P}, \mathrm{S}, \mathrm{L})$ for their measured values in both the BL direction and MD direction $(\mathrm{p}>0.05)$ (Table 2$)$.

\section{Discussion}

In our study, our hypothesis was that the primary stability of the implants placed during alveolar split osteotomy would not be affected by the size or shape of the graft particles placed between the cortical bone layers separated by split osteotomy. In order to test our hypothesis, standard width $(3 \mathrm{~mm})$ split osteotomy was performed using piezo surgery on sheep ribs and two dental implants were placed in each group. After the implantation of the dental implants, the space between the cortical bone layers was filled with small particle, large particle, and putty graft material, and the possible effects of graft materials on the resonance frequency analysis (RFA) measurements of dental implants and their primary stability were compared between the groups. Although RFA is defined as a criterion that shows the suitability of implants for the immediate loading technique, it can also be used to assess the primary stability of implants placed in atrophic areas that first require augmentation procedures [5]. There are also studies indicating that the ISQ values can be affected by the implant placement position, bone graft use, and cortical bone thickness $[7,16,17]$. Primary stability is thought to be an effective factor in the evaluation of osseointegration of dental implants [5]. Primary stability is influenced by factors such as bone quality and quantity, implant geometry, and technique of implant surgery [13]. In our study, we tried to minimize all factors that might affect primary stability in order to examine the possible pure effects of graft particles. In order to rule out the effects of bone quality and quantity, sheep ribs with thick compact bone surrounding the spongious bone with a dense trabecular web were selected in line with the classic classification of Lekholm and Zarb [15]. The absence of a significant difference between the groups and between the first and second implants for the values measured in the BL direction may indicate that the established test setup was standardized and controlled between both the first and second implants. A similar interpretation may not be made in the MD direction due to the possible effect of the graft particles. In our study, both BL and ML measurements were made in the same direction to prevent a possible change according to the measurement direction. Some researchers studied how the measurement direction affected the ISQ values of implants. Studies have shown that there is no statistically significant difference between the buccal surface with defect and the ISQ values of the lingual surface without defect [18]. In our study, it could be interpreted that there was no significant grafting between the groups in the BL direction and that the ISQ values measured in the BL direction did not differ significantly between the groups because the primary stability was only provided with inter-cortical bone surfaces. There was a statistically significant difference between the values measured in the BL direction compared with the MD direction. This finding may show that the support from the inter-cortical bone for primary stabilization is more effective in terms of ISQ than graft application. In order to eliminate the differences that may develop due to the surgical procedure, split osteotomy was performed using piezo surgery, which also helps to reduce the complications that can occur during split osteotomy. Also, the procedure is less affected by the surgeon's dexterity or the selected procedure [6-8]. The alveolar split osteotomy method used in the study was performed as described by Scipioni et al. in 1994 [4]. A suitable inter-cortical space is created by split osteotomy. This technique prevents necrosis of the outer surface of the bone cortex and allows the placement of implants and / or bone grafts [5]. In order to eliminate the effects of the implant geometry, all implants used were NobelActive implants. There are studies showing that autogenous grafts, allografts, xenografts, and synthetic materials are used to protect the bone around the implant after immediate implant placement, but none has superiority over each another [19-21]. However, xenografts can protect the alveolar bone and remain stable without dimensional change because they have osteoconductive effects and also a space-saving feature [22, 23]. According to McGlumphy and Larsen's suggestion of graft placement or directed bone regeneration when the gap between implant and bone is more than $1 \mathrm{~mm}$, deproteinized bovine bone graft containing grafts were used in our study [24]. There are studies examining the effect of bone grafts and membrane applications to achieve bone regeneration directed into the space between the bone layers that separate during alveolar split osteotomy [25-27]. In a study on dogs, it was reported that the use of grafts and/or membranes in the space between implants placed after alveolar split osteotomy prevented marginal bone loss [28]. In a pig study, the authors stated that after the application of alloplastic biphasic calcium phosphate graft and membrane around the implants placed immediately after alveolar split osteotomy, the cavity formed with the split technique was filled with bone regardless of the graft or membrane used after 6 weeks [29]. There are studies in which the cavity formed during alveolar split osteotomy was filled with xenograft or alloplastic graft materials and its 
contribution to bone formation was examined, but no study investigating the effect of these graft materials on primary stability has been found [28, 29]. In our study, it was aimed to investigate the effect of DBBM grafts on primary stabilization by imitating augmentation of the inter-cortical space formed after split osteotomy using different sized materials. According to our findings, placing deproteinized bovine-derived bone grafts with the same content but different particle sizes in the cavity had no significant effect on the primary stability of the implants. Some researchers investigated the effect of graft materials on primary stability of implants after sinus lift operation using deproteinized bovine-derived grafts with different particle sizes. According to the results of Jensen et al., small and large particles had no statistically significant effect on ISQ values [30]. However, the primary stability values of the implants were measured when implants were placed, before the sinus cavity was grafted. In that study, the effects of grafts on stability were not evaluated in terms of primary stability of implants, secondary stability of implants were evaluated after sacrifice [30]. Depending on the duration of the evaluation of stability, Jensen et al. did not consider to fully examine the effect of graft particles on ISQ values. The main evaluation is the effect of the graft particles on the ossification process, the findings of which are that small particles have a significantly higher osteoconductive effect at 6 weeks compared with large particles [30]. This finding overlaps with the hypothesis that small particles can accelerate bone formation by increasing the surface area for osteoblastic activity. Therefore, the study of Jensen et al. is thought to have measured the effect of graft particles on osseointegration, that is, secondary stabilization rather than primary stability [30]. Jensen et al. showed that graft materials with different particle sizes did not differ significantly in the quality and quantity of the new bone that formed after 12 weeks [30]. In the literature, no other study has evaluated the possible effects of graft materials with different particle sizes on ISQ values by measuring immediately after the placement of implants and immediately after the graft material has been applied. Since there is no study that can fully refer to the effects of graft particles on alveolar split osteotomy, studies that meet the criteria in this study were examined, considering that placing graft particles after implants have been implanted in our study will be methodologically similar. One study stated that placing graft particles in the cavity formed immediately after implantation following tooth extraction made a significant difference on the ISQ values of grafts in the group with initial ISQ values below 65 without grafting [23]. The averages of ISQ values in our study were below 65 in all groups; it was observed that graft applications made no significant difference compared with the control group, The lowest ISQ average value in our study was $45.7 \pm 5$ (first implant, control group M-D) and the highest value was $59.3 \pm 2.4$ (first implant, large group, BL). In general, ISQ values are thought to vary between 45-85 [5]. The data we obtained also remained in this range.

Table 1. Evaluation of primary implant stability of the first and second implants in terms of groups and distances.

\begin{tabular}{|c|c|c|c|c|c|c|}
\hline \multirow{3}{*}{ Implant } & \multirow{3}{*}{ Direction } & \multicolumn{4}{|c|}{ Primary Stability } & \multirow{3}{*}{${ }^{1} \mathbf{p}$} \\
\hline & & Group C & Group P & Group S & Group L & \\
\hline & & Mean \pm SD & Mean \pm SD & Mean \pm SD & Mean \pm SD & \\
\hline \multirow{3}{*}{ 1. Implant } & B-L & $57,5 \pm 4$ & $57,4 \pm 3,2$ & $57,4 \pm 2,8$ & $59,3 \pm 2,4$ & 0,466 \\
\hline & M-D & $45,7 \pm 5$ & $47,5 \pm 4,7$ & $47 \pm 5,1$ & $49,4 \pm 4$ & 0,381 \\
\hline & ${ }^{2} \mathrm{p}$ & $0,000^{*}$ & $0,000 *$ & $0,000 *$ & $0,000 *$ & \\
\hline \multirow[t]{3}{*}{ 2. Implant } & B-L & $58,1 \pm 2,9$ & $58,3 \pm 2,7$ & $58,4 \pm 2,2$ & $60 \pm 2,6$ & 0,348 \\
\hline & M-D & $47,5 \pm 5,1$ & $48,4 \pm 4,5$ & $48,2 \pm 4,4$ & $49,8 \pm 3,9$ & 0,708 \\
\hline & & $0,000 *$ & $0,000 *$ & $0,000 *$ & $0,000 *$ & \\
\hline${ }^{I}$ Oneway ANOVA Test & ${ }^{2}$ Student test & & & & $* p<0.05$ & \\
\hline
\end{tabular}

Table 2. Evaluation of primary implant stability of first and second implants separately in groups and distances.

\begin{tabular}{|c|c|c|c|c|}
\hline \multirow{3}{*}{ Group } & \multirow{3}{*}{ Direction } & \multicolumn{2}{|c|}{ Primary Stability } & \multirow{3}{*}{$\mathbf{p}$} \\
\hline & & 1. Implant & 2. Implant & \\
\hline & & Mean \pm SD & Mean \pm SD & \\
\hline \multirow{2}{*}{ Group C } & B-L & $57,5 \pm 4$ & $58,1 \pm 2,9$ & 0,704 \\
\hline & M-D & $45,7 \pm 5$ & $47,5 \pm 5,1$ & 0,437 \\
\hline \multirow[t]{2}{*}{ Group P } & B-L & $57,4 \pm 3,2$ & $58,3 \pm 2,7$ & 0,506 \\
\hline & M-D & $47,5 \pm 4,7$ & $48,4 \pm 4,5$ & 0,665 \\
\hline \multirow[t]{2}{*}{ Group S } & B-L & $57,4 \pm 2,8$ & $58,4 \pm 2,2$ & 0,392 \\
\hline & M-D & $47 \pm 5,1$ & $48,2 \pm 4,4$ & 0,579 \\
\hline \multirow[t]{2}{*}{ Group L } & B-L & $59,3 \pm 2,4$ & $60 \pm 2,6$ & 0,538 \\
\hline & M-D & $49,4 \pm 4$ & $49,8 \pm 3,9$ & 0,823 \\
\hline
\end{tabular}

Student t test.

\section{Conclusion}

Using ISQ values to evaluate primary stability, it was found that the different sizes of graft materials used caused no significant difference for the primary stability of the implants.
This situation can be interpreted as that the primary stability of implants taken from the inter-cortical bone layers in the BL direction may be sufficient and that an extra graft application may not contribute to primary stability. In the literature, the increase in ISQ values after bone regeneration with the use of 
grafts can be explained by the transformation of bone particles into new bone tissue. The results of our study should be supported by animal experiments and clinical studies.

\section{Approval}

For this study, no animal was killed, and fresh cow ribs of the same animal with similar properties were selected from the butcher shop. Ethical protocol is not required for this research.

\section{Conflict of Interest}

The authors declare that they have no competing interests.

\section{Acknowledgements}

This in-vitro study was performed at Okan University, Faculty of Dentistry; authors of the study are grateful to Okan University for their scientific help. We thank Ebru Osmanoglu Akyol, $\mathrm{PhD}$, for statistical review and analysis.

\section{References}

[1] McAllister, B. S. and K. Haghighat (2007). Bone Augmentation Techniques. Journal of Periodontology, 78 (3): p. 377-396.

[2] Merheb, J., et al. (2010). Influence of bony defects on implant stability. Clinical oral implants research, 21 (9): p. 919-923.

[3] Moro, A., et al. (2017). Alveolar ridge split technique using piezosurgery with specially designed tips. BioMed research international,. 2017.

[4] Scipioni, A., G. B. Bruschi, and G. Calesini (1994). The edentulous ridge expansion technique: a five-year study. International Journal of Periodontics \& Restorative Dentistry, $14(5)$.

[5] González-García, R., F. Monje, and C. Moreno (2011). Alveolar split osteotomy for the treatment of the severe narrow ridge maxillary atrophy: a modified technique. International journal of oral and maxillofacial surgery. 40 (1): p. 57-64.

[6] Stacchi, C., et al. (2013). Changes in implant stability using different site preparation techniques: twist drills versus piezosurgery. A single-blinded, randomized, controlled clinical trial. Clinical implant dentistry and related research,. 15 (2): $p$. 188-197.

[7] Vercellotti, T., S. De Paoli, and M. Nevins (2001). The piezoelectric bony window osteotomy and sinus membrane elevation: introduction of a new technique for simplification of the sinus augmentation procedure. International Journal of Periodontics \& Restorative Dentistry. 21 (6).

[8] Vercellotti, T. (2004). Technological characteristics and clinical indications of piezoelectric bone surgery. Minerva stomatologica. 53 (5): p. 207-214.

[9] Tolstunov, L., et al. (2019). Bone augmentation techniques for horizontal and vertical alveolar ridge deficiency in oral implantology. Oral and Maxillofacial Surgery Clinics. 31 (2): p. 163-191.
[10] Lioubavina-Hack, N., N. P. Lang, and T. Karring (2006). Significance of primary stability for osseointegration of dental implants. Clinical oral implants research. 17 (3): p. 244-250.

[11] Meredith, N 1998. Assessment of implant stability as a prognostic determinant. International Journal of Prosthodontics. $11(5)$.

[12] Bilhan, H., et al. (2010). Influence of surgical technique, implant shape and diameter on the primary stability in cancellous bone. Journal of oral rehabilitation. 37 (12): p. 900-907.

[13] Sennerby, L. and J. Roos (1998). Surgical determinants of clinical success of osseointegrated oral implants: a review of the literature. International Journal of Prosthodontics. 11 (5).

[14] Tanaka, K., et al. (2018). Relationship between cortical bone thickness and implant stability at the time of surgery and secondary stability after osseointegration measured using resonance frequency analysis. Journal of periodontal \& implant science. 48 (6): p. 360-372.

[15] Zarb, G. A. and T. Albrektsson (1985) Tissue-integrated prostheses: osseointegration in clinical dentistry. Quintessence Pub Co.

[16] Ersanli, S., et al. (2005). Resonance frequency analysis of one-stage dental implant stability during the osseointegration period. Journal of periodontology. 76 (7): p. 1066-1071.

[17] Östman, P.-O., et al. (2006). Resonance frequency analysis measurements of implants at placement surgery. International Journal of Prosthodontics. 19 (1).

[18] Yim, H.-j., et al. (2019) Primary stability of implants with peri-implant bone defects of various widths: an in vitro investigation. Journal of periodontal \& implant science. 49 (1): p. 39-46.

[19] Atalay, B., et al. (2013). Immediate implant placement without bone grafting: a retrospective study of 110 cases with 5 years of follow-up. Implant dentistry. 22 (4): p. 360-365.

[20] Santos, P. L., et al. (2013). Bone substitutes for peri-implant defects of postextraction implants. International journal of biomaterials. 2013 .

[21] Viswambaran, M., et al. (2014), Clinical evaluation of immediate implants using different types of bone augmentation materials. medical journal armed forces india. 70 (2): p. 154-162.

[22] Barone, A., et al. (2008). Xenograft versus extraction alone for ridge preservation after tooth removal: a clinical and histomorphometric study. Journal of periodontology. 79 (8): p. 1370-1377.

[23] Jun, S. H., et al. (2018). The influence of bone graft procedures on primary stability and bone change of implants placed in fresh extraction sockets. Maxillofacial plastic and reconstructive surgery. 40 (1): p. 8.

[24] Mc Glumphy EA, L. P. (2003). Contemporary implant dentistry, in Contemporary oral and maxillofacial surgery., E. E. Peterson LJ, Hupp JR,, Editor. Mosby: St. Louis. p. 305-342.

[25] Bassetti, M. A., R. G. Bassetti, and D. D. Bosshardt (2016). The alveolar ridge splitting/expansion technique: a systematic review. Clinical oral implants research. 27 (3): p. 310-324. 
[26] Ella, B., et al. (2014). Mandibular ridge expansion using a horizontal bone-splitting technique and synthetic bone substitute: an alternative to bone block grafting? International Journal of Oral \& Maxillofacial Implants. 29 (1).

[27] Tang, Y. L., et al. (2015). Ridge expansion alone or in combination with guided bone regeneration to facilitate implant placement in narrow alveolar ridges: a retrospective study. Clinical oral implants research. 26 (2): p. 204-211.

[28] Han, J. Y., et al. (2011). The effects of bone grafting material and a collagen membrane in the ridge splitting technique: an experimental study in dogs. Clinical Oral Implants Research. 22 (12): p. 1391-1398.
[29] Stricker, A., et al. (2014). Evaluation of a new experimental model to study bone healing after ridge expansion with simultaneous implant placement-a pilot study in minipigs. Clinical oral implants research. 25 (11): p. 1265-1272.

[30] Jensen, S. S., et al. (2015). Influence of particle size of deproteinized bovine bone mineral on new bone formation and implant stability after simultaneous sinus floor elevation: A histomorphometric study in minipigs. Clinical implant dentistry and related research. 17 (2): p. 274-285. 